\title{
Launching the Advanced Trauma Operative Management (ATOM), Course in Trinidad and Tobago
}

\author{
Jameel Ali ${ }^{*}$, Grahame Laurence ${ }^{2}$, Danielle Bince1, Curtis Legall ${ }^{3}$, Karla Georges ${ }^{3}$, \\ Marc Alkhal ${ }^{3}$, Anjan Nanan'3 , Sunildath Jugool4, Rasheed Adam5, Melrose Yearwood5, \\ Grace Herrera Fernandez', Dave Harnanan7, Dilip Dan?7, Jennifer Reece ${ }^{3}$, Anil Persad ${ }^{3}$ \\ ${ }^{1}$ University of Toronto, Toronto, Canada \\ ${ }^{2}$ Holy Spirit Hospital, Camp Hill, PA, USA \\ ${ }^{3}$ School of Veterinary Medicine, University of the West Indies, Mt. Hope, Trinidad and Tobago \\ ${ }^{4}$ Sangre Grande Hospital, Sangre Grande, Trinidad and Tobago \\ ${ }^{5}$ Eric Williams Medical Science Complex, Champs Fleurs, Trinidad and Tobago \\ ${ }^{6}$ Costa Rica Trauma Program, San Jose, Costa Rica \\ ${ }^{7}$ Department of Surgery, University of the West Indies, Mt. Hope, Trinidad and Tobago \\ Email: ^jameel.ali@unityhealth.to
}

How to cite this paper: Ali, J., Laurence, G., Bince, D., Legall, C., Georges, K., Alkhal, M., Nanan, A., Jugool, S., Adam, R., Yearwood, M., Fernandez, G. H., Harnanan, D., Dan, D., Reece, J., \& Persad, A. (2020). Launching the Advanced Trauma Operative Management (ATOM), Course in Trinidad and Tobago. Creative Education, 11, 2570-2579.

https://doi.org/10.4236/ce.2020.1112189

Received: November 6, 2020

Accepted: December 7, 2020

Published: December 10, 2020

Copyright (c) 2020 by author(s) and Scientific Research Publishing Inc. This work is licensed under the Creative Commons Attribution International License (CC BY 4.0).

http://creativecommons.org/licenses/by/4.0/

\section{(c) (i) Open Access}

\begin{abstract}
Background: The Advanced Trauma Operative Management (ATOM) course which is aimed at improving penetrating trauma management skills is very challenging to conduct. We assessed the feasibility and potential impact of ATOM training in Trinidad and Tobago through the University of Toronto Global surgery initiative and its potential for improving penetrating trauma care in this developing country. Methods: Senior General Surgical trainees were randomly assigned to participate. Other participants consisted of: an experienced international ATOM course Director, one experienced ATOM instructor, an ATOM instructor candidate, an experienced ATOM veterinary medicine technologist, 2 veterinary medicine trainees, 8 senior general surgical trainees who completed the course using the 2 student to one faculty training model. Pre and post course self efficacy scores (a measure of confidence in surgical approach) and scores on multiple choice question exams (MCQ) were compared by paired t tests. The trainees completed 5-point Likert scales to assess different components of the course. Results: The course was successfully completed locally. Mean and SD self efficacy scores improved from $55.4 \pm 18.5$ to $80.5 \pm 9.1$ and MCQ improved from $63.0 \pm 8.8$ to $82.5 \pm 9.6(p<.001)$. Rating of lectures, procedures and general assessment by Likert scores ranked the experiences as very good to excellent. Conclusions: Based on trainee course performance and their evaluations, there was signifi-
\end{abstract}


cant improvement in trauma skills, knowledge and attitude with enthusiastic support for continuing the program, to improve penetrating trauma care locally and extending this training to other parts of the Caribbean.

\section{Keywords}

Trauma Teaching, ATOM Course, ATOM in Developing Country

\section{Introduction}

The ATOM (Advanced Trauma Operative Management) course was initiated in Hartford Connecticut in 1998 through the pioneering work of Dr. Lenworth Jacobs (Jacobs, Burns, \& Kaban, 2003). The course was adopted by the American College of Surgeons Committee on Trauma in 2008 and has since been promulgated internationally (Jacobs, Burns, \& Luk, 2005) in much the same way as the Advanced Trauma Life Support Course (Ali, Adam, \& Butler, 1993; Ali \& Howard, 1993; Ali, Cohen, \& Adam, 1996) which is now well established in Trinidad and Tobago and other Caribbean islands. Interest in introducing ATOM training in Trinidad and Tobago has long been a goal of leading faculty surgeons of the University of the West Indies Mount Hope campus as well as general surgeons practising in other hospitals in this country. These surgeons communicated their interest to the American College of Surgeons, an interest that was heightened by the establishment and activities of the Trinidad and Tobago American College of Surgeons chapter. Permission to initiate the course was approved by the American College of Surgeons in 2019 recognizing the high local incidence of penetrating trauma, being among the highest in the world (Haagsma, Greetz, \& Boliger, 2016) with resulting high mortality particularly among the young as it is in most other parts of the world. There is a well established Advanced Trauma Life Support program in Trinidad and Tobago where the first program outside North America was established in January 1986. However, in spite of the high incidence of Trauma in this part of the world there is no formal postgraduate Trauma training program in Trinidad and Tobago.

Programs such as the ATOM course have been introduced throughout the world because of several factors which limit live trauma patient operative experience among surgical trainees. These factors include shortening of residency training programs, a decrease in frequency of on call duties and an increasing trend towards nonoperative management of trauma. Apart from the ATOM course, other programs aimed at addressing this deficiency in clinical postgraduate trauma training include the Definitive Surgical Trauma Care (DSTC) course and the Advanced Surgical Skills for Exposure in Trauma (ASSET) course (Ali, Sorvari, \& Haskin, 2011).

One of the major reasons that this program has not been widely adopted in developing countries is the very challenging resource requirement particularly in the maintenance and survival of the animal model in withstanding the severe 
injuries and the long duration of the many procedures. A major goal of launching this program in Trinidad and Tobago was to determine the feasibility of conducting this program in such environments so that the program may be adopted more widely internationally because of its potential and dire need for improving outcome in penetrating injuries in such countries.

These programs such as the Advanced Trauma Life Support course have been demonstrated to improve not only the knowledge and skills required for clinical trauma management but most importantly improving survival in both blunt and penetrating injuries.

In accordance with established American College of Surgeons Committee on Trauma ATOM promulgation guidelines, a site visit and report to the ATOM subcommittee by National ATOM faculty was completed and approved. Also approved by the subcommittee was the required Animal Care Ethics committee protocol. This was followed by training of faculty for the inaugural ATOM course in Trinidad and Tobago.

We describe the conduct of this inaugural course, including data from the course and its potential application in Trinidad and Tobago.

\section{Methods}

\section{Faculty Preparation:}

The traditional ATOM course utilizing the 1 student to 1 faculty model has stood the test of time as a very effective teaching tool and is still the generally recognized model for teaching the course successfully as documented widely in the literature (Ali, Ahmed, \& Jacobs, 2003). In 2013, at the Toronto ATOM site we sought and were given permission by the ATOM Committee to conduct the course using a 2 student to 1 faculty model with on site outside observation and participation by a senior ATOM National faculty member to gather data on this model and report back to the ATOM committee. This model proved very educationally successful as reported elsewhere (Ali, Sorvari, \& Henry, 2013). With our Toronto site being allowed to use this 2 student to 1 faculty model under strict ATOM Committee guidelines including an approved experienced 2 student to 1 ATOM faculty Course Director. Our intent was to use this 2 student to 1 faculty model for the Trinidad and Tobago Course which required the qualified 2 student to 1 faculty Course Director and detailed preparation of the faculty to ensure adherence to ATOM 2 to 1 model course guidelines.

This preparation began with 2 veterinary medical staff from Trinidad and Tobago attending and participating in our 2 student to 1 faculty model for 12 participants at our Toronto site and under the close tutelage of our most senior Veterinary laboratory technologist from the Toronto site focusing on meticulous vascular access technique and general care of the animals. This same veterinary laboratory technologist travelled to Trinidad for the inaugural course to supervise the proper conduct of vascular access and care of the animals. The other faculty members, apart from the experienced ATOM 2 to 1 Course Director con- 
sisted of one senior ATOM instructor from Philadelphia and one ATOM instructor candidate from Trinidad and Tobago.

\section{Animal Preparation:}

One of the most important and challenging features of the ATOM course is ethical humane care of the animals with constant hemodynamic (arterial blood pressure and electrocardiogram) monitoring, core temperature, end tidal $\mathrm{CO}_{2}$, $\mathrm{O}_{2}$ saturation and arterial blood gases (as frequently as required), with constant monitoring of the level of general anesthesia by the veterinary medical staff/ anesthetist to ensure that the animals experience no pain ensuring and maintaining the core temperature by using a constant supply of warm intravenous fluids.

The animal care protocol was reviewed and approved by the University of the West Indies Campus Ethics Committee (reference number-CEC1173/06/19), submitted to and approved by the ATOM Committee of the American College of Surgeons. The protocol includes the following:

Source and characteristics of the animals: Female/Landrace pigs, weighing 55 - $60 \mathrm{Kgm}$ obtained from the University of the West Indies Field Station, transported to the School of Veterinary Medicine 2 days prior to the course for acclimatization and housed in individual animal stalls. They were fasted on the morning of the course, premedicated in their individual pens using Xylazine (Xylazin, Dutch Farm, Holland) at $2 \mathrm{mg} / \mathrm{kg}$ IM and Ketamine (Ketamin, Dutch Farm, Holland) at $20 \mathrm{mg} / \mathrm{kg}$ IM then transported to the surgical theatre.

Instrumentation: Temporary intravenous catheter in a marginal ear vein replaced by a secured Jugular vein catheter for intravenous infusion; an inflatable blood pressure pump used to regulate rate of IV infusion; pulse oximeter probe on the tongue; placement of ECG conductive pads on the shaved dorsal and plantar aspects of the fore and hind limbs; probes connected to a multi parameter vital signs monitor (Cardiocap/5Datex-Ohmeda, General Electric, Finland); intubated in the lateral recumbency position with a cuffed, $7.5 \mathrm{~mm}$, endotracheal tube and connected to a circle circuit rebreathing anesthesia machine (VIP 3000 Matrix, Midmark); common carotid artery dissected, secured, cannulated and connected to an invasive blood pressure monitor.

The animals were maintained with Isoflurane/ oxygen and an intermittent positive pressure ventilator was used to regulate respiration. Intravenous fluid plastic bags were kept in a constantly flowing warm fluid bath to maintain temperature of the infused fluid. Resuscitative drugs such as adrenaline, dobutamine, anti-arrhythmics and a defibrillator were also available.

After all the surgical procedures required in the course were satisfactorily completed, optional extra procedures such as atrio-caval shunt placement were practiced by the participants.

At the end of the procedures including the optional ones, the animals that did not succumb during the additional procedures were euthanized by intracardiac $10 \%$ potassium chloride injection. 
Course Participants (trainees):

The final year general surgery residents in the program were invited to apply for the course and the first 8 were accepted to take the course after instructions to register with the ATOM committee and complete the precourse questionnaires and reading material.

Course Schedule:

The course was conducted on August 9-11 2019.

Day One afternoon (August $9^{\text {th }}$ ): the lectures were delivered by the Course director, instructor candidate and fully trained ATOM course instructor.

Second day am (August $10^{\text {th }}$ )-There were 2 participants with one designated instructor supervised by the Course Director (a recognized 2 to 1 ATOM model course director) with one 55 - $60 \mathrm{kgm}$ anesthetized pig. Both participants performed as operating surgeon and surgical assistant in 2 of each operation switching roles in between.

The procedures were completed over 4 hours with 2 of each injury-so each participant was involved in 2 of each procedure as an assistant and as a performing surgeon.

The "non bleeding" injuries were created and treated first in order to start with least stressful procedures: laparotomy, small bowel, duodenum, stomach, ureter, bladder, diaphragm.

The bleeding injuries were then created and treated: renal hematoma, renal laceration, pancreas, spleen and liver, leaving the IVC and Cardiac injuries for the 3rdphase because they were the ones with the likelihood of the largest blood loss.

Third phase: 2 separate IVC and 2 separate cardiac injuries were created and treated by the 2 participants as operating surgeon and surgical assistant.

This same rotation was followed for 2 more participants with one animal in the afternoon of the second day (August 10th).

The same sequence for 2 participants in the morning and 2 in the afternoon were followed on Day 3. (August $11^{\text {th }}$ ).

Over the 2 operating days, 8 participants were trained using 4 animals.

Each group completed the post course questionnaires and evaluations before leaving the course site after feedback.

An experienced Course Coordinator from other medical courses at the centre was also trained during the course as an ATOM Course Coordinator candidate.

The lectures were conducted in the lecture theatre of the School of Veterinary Medicine and the surgical procedures were conducted in the large animal operating suite of the school.

\section{Analysis of Data:}

MCQ examination: Pre and post course mean MCQ scores (\%) were compared by Paired " $\mathrm{t}$ " tests

SELF-EFFICACY scores (\%) have been validated as a reproducible and reliable measure of confidence in operative approach in many studies (Bandura, 1977). The mean self efficacy scores were also compared pre and post course by 
paired " $t$ " tests.

The self-efficacy scores represent one of the few parameters that are capable of measuring an important but frequently difficult and evasive quality for assessing the "subjective" element of confidence in the operating room. A p-value of .05 was considered statistically significant for these paired " $t$ " tests.

Rating of lectures and Procedures was performed by the trainees using a Likert scale:

1) poor, 2) fair, 3) good, 4) very good and 5) excellent)

The participants also rated the educational value of the procedures and the course in general as well as making general comments and suggestions about the course on the evaluation forms.

\section{Results}

All 8 participants successfully completed all the course procedures in live animals.

Three of the animals succumbed during the additional optional procedures but survived for all of the regular course procedures and the $4^{\text {th }}$ animal which survived all the optional and regular course procedures was euthanized at the end of the course by intracardiac injection of $10 \% \mathrm{KCl}$ according to protocol.

Mean pre and post course MCQ scores and Self Efficacy scores with Standard deviations and $\mathrm{p}$ values are shown in Table 1 where it is seen that there was a statistically significant improvement in both these mean scores after the course.

Rating of the lectures from 1) poor, 2) fair, 3) good, 4) very good and 5-excellent is shown in Table 2 with the number of participants(n) out of 8 for each rating. The laparotomy lecture had an excellent rating by all 8 participants and the students rated the lectures at least very good.

Rating of the procedures:

All 8 participants rated duodenum, bladder, vena cava and cardiac procedures as 5 .

The remaining procedures (stomach, kidney, ureter, spleen, laparotomy, small bowel, liver, pancreas, diaphragm) were rated 5 by 2 participants and 4 by 6 participants.

When asked for choosing the best overall learning experience procedure, IVC was chosen by 6 participants and kidney by 2 participants.

Table 1. Mean pre and post course Self Efficacy and MCQ scores with Standard deviations.

\begin{tabular}{lcc}
\hline \multicolumn{3}{c}{ Self Efficacy Scores: } \\
\hline Pre course & \multicolumn{2}{c}{ Post Course } \\
$55.4(18.5)$ & $80.5(9.1)$ & $p=.001$ \\
& MCQ exam scores: & \\
Pre course & Post Course & \\
$63.0(8.8)$ & $82.5(9.6)$ & \\
\hline
\end{tabular}


Table 2. Evaluation of Lectures and Procedures were conducted using a Likert scale-: (1) poor, 2) fair, 3) good, 4) very good, 5) excellent). Lectures were rated.

\begin{tabular}{ccc}
\hline Lectures & $\mathrm{n}$ & rate \\
\hline Laparotomy & 8 & 5 \\
Liver & 6 & 4 \\
Spleen/Diaphragm & 2 & 5 \\
Pancreas/Duodenum & 6 & 4 \\
Genito/Urinary & 2 & 3 \\
Cardiac/Vascular & 6 & 4 \\
& 2 & 3 \\
& 4 & 4 \\
& 4 & 3 \\
\hline
\end{tabular}

\section{Comments:}

All 8 participants recommended that the course become an integral continuing part of their training in General Surgery.

Six of the 8 participants felt that the program is ideal during their final year while the other 2 suggested that the course should be offered in the penultimate year allowing them more time to practice what they learnt in the penultimate year during their final year of training.

On a scale of 1) strongly disagee, 2) disagree, 3) neutral, 4) agree, 5) strongly agree, all students strongly agreed that the 2 student model was excellent because they were exposed to the same injury twice, once as an operating surgeon and also as a surgical assistant because these 2 roles require different skills in the operating room with a different type of learning experience.

On the same Likert scale of $1-5$ as in the above paragraph, ratings were 4 to 5 for the objectives being met and improvement in clinical and judgement skills as well as improvement in confidence.

While recognizing that the ideal training would be in live patients, the live animal model was considered an excellent alternate by all participants for this type of training.

\section{Limitations of the Study}

The major limitation of the study is the small number of participants trained in this course.

Ideally, a larger number of participants would engender greater statistical power and confidence in our conclusion. and could be undertaken with availability of more resources such as ventilators, laboratory space, equipment and staffing. There is room for planning this in future courses. However, to the extent that we were examining possibilities of introducing the program in this poor 
resourced environment, the data suggest enthusiasm for the future of ATOM in Trinidad and Tobago and the statistically significant results with minimal data points is encouraging.

Further work involving larger numbers of candidates is planned to more strongly support introducing such training programs. The knowledge gained from this small set of candidates however, is important in guiding the way for generating more data in the future.

We made statements about the perceived educational superiority of the 2 students to 1 faculty model but a more definitive assessment in this regard would require a control group of students trained on the 1 student to 1 faculty model. Such a comparison was reported in our previous publication with similar outcome using a control group (Ali, Sorvari, \& Henry, 2013).

Although there was a trend to suggest that the course is better conducted in the final year of training, the numbers were again too small to definitively draw this conclusion.

The Likert scores methodology is very subjective and requires caution in interpretation as with all Likert scores without a control group. However, we are probably safe in cautiously concluding based on these data that the course was highly rated and uniformly successful as perceived by the participants and it is probably safe to conclude that the future of ATOM course promulgation in this country is promising.

\section{Conclusion and Future Directions}

This inaugural ATOM course was successfully completed in Trinidad and Tobago meeting internationally recognized standards. All participants met cognitive and skills expectations of the course. The course was very highly rated by all participants whose confidence in surgical approaches to these injuries improved as measured by the pre and post course self-efficacy scores. Faculty is now available at the Trinidad site to conduct future courses. For the 2 to 1 model an experienced approved 2 to 1 model ATOM Course Director will be necessary as required by ATOM guidelines. A local ATOM Course Director with good proven leadership and Team relationship skills will need to be recommended after being critiqued as a satisfactory Course director candidate and meeting other ATOM guidelines.

There was unanimous enthusiastic support for continuing ATOM training of more faculty and surgical trainees, offering the course to all senior general surgery residents eventually in order to better meet the challenge of treating the very large population of patients suffering penetrating torso injuries in Trinidad and Tobago with the prospect of extending this training to other Caribbean centres.

Trinidad and Tobago have well established Advanced Trauma Life Support (ATLS), Prehospital Trauma Life support (PHTLS), Rural Trauma Team Development (RTTDC) and Trauma Evaluation and Management (TEAM) courses (Ali, Adam, \& Gana, 1993; Ali, Ali, \& Adam, 2015; Ali, Cherry, \& Williams, 
2005) which they have successfully promulgated to other Caribbean Islands. These have impacted positively on development of Ambulance systems and training of prehospital personnel in this country.

Faculty involved in these programs should consider establishing training units in the lead institutions with a focus on trauma education and care. These units should be led by staff interested and trained in trauma management, encouraging trauma research and education. Formal rotation of trainees on these units with multi institutional trauma rounds conducted through electronic transmission modes weekly or biweekly on a rotation basis among the institutions could be conducted to enhance trauma care and education. Trauma teams with designated trauma team leaders would facilitate coordination of trauma care in the lead institutions as is done in trauma training programs in other countries. There is also the opportunity for local trainees to present trauma papers in the international annual resident trauma papers competition held and sponsored by the American College of Surgeons if resident papers are chosen to represent the Region (Region 14 of ACS Committee on Trauma). Consideration should be given to reinstitute participation in the International Tele trauma rounds held weekly.

The above activities can be realized in this country which sorely needs emphasis in trauma education because of the major trauma mortality problem in Trinidad and Tobago. Such activities represent a natural spinoff from not only this recently completed ATOM course but also the already established role in trauma education internationally through the other trauma training courses conducted locally. In this way, the trauma training courses would not be just isolated courses but part of a more comprehensive program geared towards improving trauma education and care generally in this country.

\section{Acknowledgements}

The ACS Committee on Trauma staff, particularly Dr. Jody Kaban, Surgical Skills chair, Dr. Sharon Henry, International Trauma Education program chair, Shana Kachaochana Trauma Education Program Coordinator. University of Toronto: Professor of Global Surgery Program Dr. Lee Errett and Dr James Rutka, Professor and Chair Dept of Surgery. AA Laquis, local representatives for Johnson and Johnson: Dirk Cabral, Solange De Vignes, Kristal Seepaulsingh and Nicole Huggins. Mt Hope School of Veterinary Medicine support staff: Mrs Ruth Seerattan and Sujal Bedassie, Roger Malcolm, vivarium technical staff.

\section{Conflicts of Interest}

The authors declare no conflicts of interest regarding the publication of this paper.

\section{References}

Ali, J., \& Howard, M. (1993). The Advanced Trauma Life Support Program in Manitoba. A 5-Year Review. Canadian Journal of Surgery, 36, 181-183. 
Ali, J., Adam, R., \& Butler, A. K. (1993). Trauma Outcome Improves Following the Advanced Trauma Life Support (ATLS) Program in a Developing Country. The Journal of Trauma, 34, 890-899. https://doi.org/10.1097/00005373-199306000-00022

Ali, J., Adam, R., \& Gana, T. (1993). Impact of the Prehospital Trauma Life Support Program in Trinidad and Tobago. West Indian Med Journal, 47, 102-104.

Ali, J., Ahmed, N., \& Jacobs, L. M. (2003). The Advanced Trauma Operative Management Course in a Canadian Residency Program. Canadian Journal of Surgery, 1, 185-189.

Ali, J., Ali, E., \& Adam, R. (2015). Is There a Role for the Rural Trauma Team Development Course (RTTDC) in Trinidad? Caribbean Medical Journal, 77, 17-18.

Ali, J., Cherry, R., \& Williams, J. I. (2005). Trauma Base Knowledge and the Effect of the Trauma Evaluation and Management (TEAM) Program among Senior Medical Students in 7 Countries. The Journal of Trauma, 59, 43-48. https://doi.org/10.1097/01.TA.0000171461.12129.10

Ali, J., Cohen, R., \& Adam, R. (1996). Attrition of Cognitive and Trauma Management Skills after the ATLS Program. The Journal of Trauma, 40, 860-866.

https://doi.org/10.1097/00005373-199606000-00002

Ali, J., Sorvari, A., \& Haskin, D. (2011). Potential Role of the ASSET (Advanced Surgical Skills for Exposure in Trauma) Course in Canada. The Journal of Trauma, 71, 1491-1493. https://doi.org/10.1097/TA.0b013e3182318053

Ali, J., Sorvari, A., \& Henry, S. (2013). The Advanced Trauma Operative Management Course-A Two Student to One Faculty Model. Journal of Surgical Research, 184, 551555. https://doi.org/10.1016/j.jss.2013.03.019

Bandura, A. (1977). Self Efficacy. Toward a Unifying Theory of Behavior Change. Psychological Review, 84, 191-215. https://doi.org/10.1037/0033-295X.84.2.191

Haagsma, J. A., Graetz, N., \& Bolliger, I. (2016). The Global Burden of Injury: Incidence, Mortality, Disability-Adjusted Life Years and Time Trends from the Global Burden of Disease Study 2013. Injury Prevention, 22, 3-18.

https://doi.org/10.1136/injuryprev-2015-041616

Jacobs, L. M., Burns, K. J., \& Kaban, J. M. (2003). Development and Evaluation of the Advanced Trauma Operative Management Course. The Journal of Trauma, 55, 471-479. https://doi.org/10.1097/01.TA.0000059445.84105.26

Jacobs, L. M., Burns, K. J., \& Luk, S.S. (2005). Advanced Trauma Operative Management Course Introduced to Surgeons in West Africa. Bulletin of the American College of Surgeons, 90, 9-14. 